\title{
Effects of Huge Earthquakes on Earth Rotation and the length of Day
}

\author{
Changyi Xu, Wenke Sun *, and Xin Zhou \\ Key Laboratory of Computational Geodynamics, Graduate University of Chinese Academy of Sciences, \\ Beijing, China
}

Received 27 February 2012, accepted 16 January 2013

\begin{abstract}
We calculated the co-seismic Earth rotation changes for several typical great earthquakes since 1960 based on Dahlen's analytical expression of Earth inertia moment change, the excitation functions of polar motion and, variation in the length of a day $(\Delta \mathrm{LOD})$. Then, we derived a mathematical relation between polar motion and earthquake parameters, to prove that the amplitude of polar motion is independent of longitude. Because the analytical expression of Dahlen's theory is useful to theoretically estimate rotation changes by earthquakes having different seismic parameters, we show results for polar motion and $\triangle \mathrm{LOD}$ for various types of earthquakes in a comprehensive manner. The modeled results show that the seismic effect on the Earth's rotation decreases gradually with increased latitude if other parameters are unchanged. The Earth's rotational change is symmetrical for a $45^{\circ}$ dip angle and the maximum changes appear at the equator and poles. Earthquakes at a medium dip angle and low latitudes produce large rotation changes. As an example, we calculate the polar motion and $\Delta$ LOD caused by the 2011 Tohoku-Oki Earthquake using two different fault models. Results show that a fine slip fault model is useful to compute co-seismic Earth rotation change. The obtained results indicate Dahlen's method gives good approximations for computation of co-seismic rotation changes, but there are some differences if one considers detailed fault slip distributions. Finally we analyze and discuss the co-seismic Earth rotation change signal using GRACE data, showing that such a signal is hard to be detected at present, but it might be detected under some conditions. Numerical results of this study will serve as a good indicator to check if satellite observations such as GRACE can detect a seismic rotation change when a great earthquake occur.
\end{abstract}

Key words: Earth rotation, Earthquake, Polar motion, Change in length of day

Citation: Xu, C., W. Sun, and X.Zhou, 2013: Effects of huge earthquakes on Earth rotation and the length of day. Terr. Atmos. Ocean. Sci., 24, 649-656, doi: 10.3319/TAO.2013.01.16.02(TibXS)

\section{INTRODUCTION}

An array of geophysical phenomena can cause Earth's rotation to change: post glacial rebound, atmospheric angular momentum change, the interaction between seawater and atmosphere, and so on (Lambeck 1980; Chao et al. 1987; Trupin et al. 1992). Those geophysical changes accompanied with density variation excite polar motion and causes a change in the day length $(\Delta \mathrm{LOD})$. Huge earthquakes can cause polar motion and $\triangle \mathrm{LOD}$ because of the redistribution of the Earth's internal density. Mansinha and Smylie (1967) described the problem related to the Chandler wobble excited by large earthquakes. They calculated the change of

\footnotetext{
* Corresponding author

E-mail:sunw@gucas.ac.cn
}

the Earth's inertia tensor and direction of Earth's axis of rotation. Dahlen $(1971,1973)$ derived an analytical expression (called Dahlen's theory) of the Earth's inertia tensor parameter change and showed the Earth's rotation change as a result of earthquakes; the modeled results indicate that the 1960 Chile earthquake $\left(\mathrm{M}_{\mathrm{w}} 9.5\right)$ produced a polar motion change with a magnitude of 10.2 mas in the direction of $110^{\circ} \mathrm{E}$. The 1964 Alaska earthquake $\left(\mathrm{M}_{\mathrm{w}} 9.2\right)$ excited a polar motion change with a magnitude of 4.8 mas in the direction of $193^{\circ} \mathrm{E}$. Except the Dahlen's theory, some researchers developed different methods to compute co-seismic Earth rotation changes. O'Connell and Dziewonski (1976) used the free oscillation method to calculate the polar motion caused by earthquakes. Smith (1977) computed the rotation change 
induced by earthquakes from 1960 using different dislocation models; the results agree well with Dahlen's results. Lambeck (1980) showed that $\Delta$ LOD caused by the 1960 Chile earthquake $\left(\mathrm{M}_{\mathrm{w}} 9.5\right)$ was $15 \mu \mathrm{s}$ and that the change induced by 1964 Alaska event $\left(M_{w} 9.2\right)$ was $3 \mu$ s. Chao and Gross (1987) computed changes in the Earth's rotation and low-degree gravitational field induced by earthquakes using a normal mode method. They concluded that the earthquake excited $\triangle \mathrm{LOD}$ is about two orders smaller than the accuracy of modern geodesy about $0.1 \mathrm{~ms}$ and that the Earth's rotation is speeding up. Degryse and Dehant (1996) presented a polynomial approximation of depth functions to complete the theory of Dahlen $(1971,1973)$. Gu (1996) using Dahlen's elastic dislocation method, computed polar motion and $\triangle \mathrm{LOD}$ caused by earthquakes occurring over 1977 - 1994 and derived a trend of shortening LOD. Gross and Chao (2006) calculated co-seismic effects caused by the 2004 Sumatra earthquake $\left(M_{w} 9.0\right)$, noting that the polar motion is 2.32 mas in the direction of $127^{\circ} \mathrm{E}$, and that LOD was shortened by $6.8 \mu \mathrm{s}$. Nilson et al. (2010) computed the co-seismic rotation change caused by the 2010 Chile earthquake $\left(M_{w}\right.$ 8.6) based on Dahlen's method and compared the theoretical results with that observed by VLBI (Very long Baseline Interferometry). They reported that the polar motion is about $2.5-3.0$ mas, and that the LOD change is about $0.3 \mu$ s. Gross (2010) also computed the co-seismic rotation change using the normal mode method. His results show that the coseismic LOD change is about $1.26 \mu$ s. Gross (2010) and Nilson et al. (2010) used different dislocation models and computing methods. Their results are somewhat different. When the 2011 Great East Japan earthquake $\left(M_{w}\right.$ 9.0) occurred, Gross (2011) estimated the co-seismic effect. His results show that the earthquake shortened LOD by $1.8 \mu$ s. Kobayashi and Heki (2012) reported a polar shift toward $140^{\circ} \mathrm{E}$.

Results of those studies indicate clearly that the rotation change has a direct relationship with an earthquake's magnitude. However, to date, no report has described an investigation of the relation between earthquake parameters and rotation changes. Some questions remain open, such as where and how an earthquake produces the largest polar motion change and $\triangle$ LOD. In this paper, we adopt Dahlen's method to compute the co-seismic earth rotation change. The purpose of this study is twofold: to study the effect of earthquake parameters upon the earth's rotation change to find a general property of seismic-excited rotation change, using Dahlen's theory due to its simplicity; and, to observe its accuracy when it is used for a single mean fault and more detailed fault slip distribution. For these purposes, we conducted numerical simulations first to test and verify Dahlen's dislocation theory; then we examined how earthquake parameters affect rotation changes. Finally we investigated the accuracy of the Dahlen's theory by conducting a case study of the 2011 Great East Japan earthquake $\left(M_{w} 9.0\right)$.

\section{THEORY AND CALCULATION METHOD OF CO-SEISMIC EARTH ROTATION CHANGE}

To elucidate the effects of earthquake parameters on rotation change, Dahlen's elastic dislocation theory is used to compute co-seismic rotation change. This method yields analytical expressions of the Earth's inertial moment which consist of functions of earthquake parameters and which are convenient to use to ascertain effects of different earthquake parameters on polar motion and $\triangle \mathrm{LOD}$. The method uses a SNREI (spherical, non-rotating, elastic, isotropic) Earth model and assumes the epicenter as a point source. We calculate polar motion and change in LOD along with evaluation of changes in Earth inertia moments, using the excitation function of polar motion and $\Delta$ LOD.

\subsection{Changes in the Inertial Moments of the Earth}

Changes in the Earth's inertial moment components induced by earthquakes can be expressed as the following analytical formulas based on Dahlen's $(1971,1973)$ elastic dislocation theory. The coordinates are chosen such that the $\mathrm{x}, \mathrm{y}$ and $\mathrm{z}$ axes point to the $0^{\circ}$ (Greenwich) Meridian, the $90^{\circ} \mathrm{E}$ Meridian and the North pole, respectively. The origin is placed at the centre of mass of the Earth.

$$
\begin{aligned}
& \Delta I_{13}=M_{0} \sum_{i=1}^{3} \Gamma_{i}(h) g_{i}(\phi, \lambda, \delta, \alpha, \theta) \\
& \Delta I_{23}=M_{0} \sum_{i=1}^{3} \Gamma_{i}(h) h_{i}(\phi, \lambda, \delta, \alpha, \theta) \\
& \Delta I_{33}=M_{0} \sum_{i=1}^{3} \Gamma_{i}(h) j_{i}(\phi, \lambda, \delta, \alpha, \theta)
\end{aligned}
$$

Therein, $\Delta I_{i j}(i j=13,23,33)$ signifies the change in inertia moments. $M_{0}$ denotes the seismic moment, and $M_{0}=$ $\mu(r) \Delta v A \cdot \mu(r)$ is the elastic shear modulus at the epicenter. Also, $\Delta v$ is the dislocation; $A$ stands for the fault area. $\Gamma_{i}(h)$, which represents the function of the depth of earthquake epicenter $h$, depends on changes of the elastic modulus and density. $g_{i}, h_{i}, j_{i}$ respectively stand for functions of latitude $\phi$, longitude $\lambda$, azimuth angle $\delta$, dip angle $\alpha$ and strike angle $\theta$. We assume that $\alpha$ is measured counterclockwise from the North Pole and that $\lambda$ is measured counterclockwise from horizontal direction on the fault plane.

To complete the Dahlen theory $(1971,1973)$, Degryse and Dehant (1996) derived the expressions of $\Gamma_{i}(h)$ in the form of polynomial approximations.

$$
\begin{aligned}
\Gamma_{1}(h)= & 0.549+5.981 \times 10^{-4} h \\
\Gamma_{2}(h)= & -1.946+0.001 h+2.738 \times 10^{-7} h^{2} \\
& -6.944 \times 10^{-10} h^{3}
\end{aligned}
$$




$$
\begin{aligned}
\Gamma_{3}(h)= & -0.050+0.002 h+2.199 \times 10^{-5} h^{2} \\
& -9.246 \times 10^{-8} h^{3}+2.667 \times 10^{-10} h^{4} \\
& -1.063 \times 10^{-12} h^{5}+2.785 \times 10^{-15} h^{6} \\
& -3.426 \times 10^{-18} h^{7}+1.560 \times 10^{-21} h^{8}
\end{aligned}
$$

Some variables used therein, $g_{i}, h_{i}, j_{i}$, are defined as shown below.

$$
\begin{aligned}
& g_{1}=a_{1} \sin 2 \phi \cos \lambda-a_{2} \cos \phi \sin \lambda \\
& g_{2}=a_{3} \sin 2 \phi \cos \lambda \\
& g_{3}=a_{4} \cos 2 \phi \cos \lambda+a_{5} \sin \phi \sin \lambda \\
& h_{1}=a_{1} \sin 2 \phi \sin \lambda+a_{2} \cos \phi \cos \lambda \\
& h_{2}=a_{3} \sin 2 \phi \sin \lambda \\
& h_{3}=a_{4} \cos 2 \phi \sin \lambda-a_{5} \sin \phi \cos \lambda \\
& j_{1}=3 a_{1} \cos ^{2} \phi \\
& j_{2}=a_{3}\left(\frac{3}{4} \sin ^{2} \phi-\frac{1}{4}\right) \\
& j_{3}=-\frac{3}{2} a_{4} \sin ^{2} \phi
\end{aligned}
$$

Furthermore, the following definitions are used.

$$
\begin{aligned}
& a_{1}=\sin 2 \alpha \sin \delta \cos \theta+\frac{1}{2} \cos 2 \alpha \sin 2 \delta \sin \theta \\
& a_{2}=\sin 2 \alpha \sin 2 \delta \sin \theta-2 \cos 2 \alpha \sin \delta \sin \theta \\
& a_{3}=-\sin 2 \delta \sin \theta \\
& a_{4}=\cos \alpha \cos \delta \cos \theta-\sin \alpha \cos 2 \delta \sin \theta \\
& a_{5}=\sin \alpha \cos \delta \cos \theta+\cos \alpha \cos 2 \delta \sin \theta
\end{aligned}
$$

For given earthquake parameters, Eqs. (1) - (10) are used to compute the change in inertial moments.

\subsection{Excitation of Polar Motion and $\Delta L O D$}

The change of the Earth's inertia tensor parameters engenders the Earth's inertial moment change. Subsequently, the Earth's rotation parameters change which alters Earth's rotational change with the following relationship outlined below.

According to the theory of Earth rotation, the excitation of polar motion caused by earthquakes can be expressed as the function of the principal moment of the Earth's inertia and inertia tensor components (Wahr 1982; Gross 1986).

$$
\psi=\frac{1.61}{C-A}\left(\Delta I_{13}+i \Delta I_{23}\right)
$$

In that equation, $A$ and $C$ are the equator and polar principal inertia moments of the Earth: $A=8.0177 \times 10^{37} \mathrm{kgm}^{2}$, and $C=8.0438 \times 10^{37} \mathrm{kgm}^{2}$.

The $\triangle$ LOD produced by earthquakes can be derived using the following expression (Gross 1986).

$\Delta \mathrm{LOD}=\frac{\mathrm{LOD}}{C_{m}} \Delta I_{33}$

Therein, $C_{m}$ is the polar principal inertia moment of the mantle; the fluid core is decoupled from the elastic mantle on a short time scale. $C_{m}=7.1242 \times 10^{37} \mathrm{kgm}^{2}, \mathrm{LOD}=86400 \mathrm{~s}$. Therefore, we can calculate the Earth's inertia tensor components as $\Delta I_{13}, \Delta I_{23}, \Delta I_{33}$ in accordance with Eqs. (1) - (3). Then, we take the three components into Eqs. (11) and (12). We can obtain the co-seismic polar motion and $\triangle \mathrm{LOD}$.

\subsection{Co-Seismic Polar Motion and $\triangle$ LOD Since 1960}

To verify the theory presented above, we compute coseismic rotation change of typical earthquakes in this section, and compare the results with previous ones. For this purpose, we choose the 1960 Chile earthquake $\left(M_{w} 9.5\right)$, 1964 Alaska earthquake $\left(M_{w} 9.2\right), 1977$ Sumba earthquake $\left(M_{w} 8.3\right), 1985$ Mexico earthquake $\left(M_{w} 8.0\right)$, and the 2004 Sumatra earthquake $\left(M_{w} 9.0\right)$. First, we compute the change in inertia moments from Eqs. (1) - (3) with consideration of the same seismic parameters for each earthquake above. Then we compute the co-seismic Earth rotation change through the relation of Eqs. (11) and (12). Table 1 presents numerical results and comparison with previous ones, and Table 2 lists the earthquake parameters used in these studies.

Table 1 shows that results obtained using different methods agree well. A difference appears in $\triangle$ LOD because the sensitivity of depth functions depends on earthquake parameters and the accuracy of different methods. When an earthquake occurs, the mass migration and mass redistribution at a global scale depend strongly on the seismic mechanism, but the present fault model can not give the accurate epicenter depth and the distribution of density change. A great difference exists between slip vectors of different fault models which cause the uncertainty of earthquake parameters. They engender the error of calculating $\triangle$ LOD. However, the present methods of co-seismic rotation change are computed by either dislocation theory or normal mode method, so that it is natural to see the difference among the results.

\subsection{Independence of Polar Motion from Longitude}

Polar motion caused by an earthquake mainly affects the amplitude and the direction. Because the direction depends on the coordinate system and the earthquake location, 
Table 1. Polar motion and $\Delta$ LOD induced by huge earthquakes after 1960 and comparison of different results.

\begin{tabular}{|c|c|c|c|c|}
\hline \multirow{2}{*}{ Events } & \multicolumn{2}{|c|}{ Polar motion } & \multirow{2}{*}{$\Delta \mathrm{LOD}(\mu \mathrm{s})$} & \multirow{2}{*}{ Author } \\
\hline & amplitude (mas) & Direction $\left({ }^{\circ}\right)$ & & \\
\hline \multirow{3}{*}{$\begin{array}{l}\text { Chile }\left(M_{w} 9.5\right) \\
(1960.05 .22)\end{array}$} & 21.5 & 111.78 & 10.76 & Gu 1996 \\
\hline & 22.6 & 115 & -8.4 & Chao and Gross 1987 \\
\hline & 24.9 & 112 & 4.97 & This study \\
\hline \multirow{3}{*}{$\begin{array}{l}\text { Alaska (M 9.2) } \\
(1964.03 .28)\end{array}$} & 8.08 & 203.75 & 5.9 & Gu 1996 \\
\hline & 7.5 & 198 & 6.8 & Chao and Gross 1987 \\
\hline & 8.52 & 201.9 & 1.66 & This study \\
\hline \multirow{3}{*}{$\begin{array}{l}\text { Sumba }\left(M_{w} 8.3\right) \\
(1977.08 .19)\end{array}$} & 0.19 & 160 & 1.05 & Gu 1996 \\
\hline & 0.21 & 116.89 & 0.33 & Chao and Gross 1987 \\
\hline & 0.45 & 151.9 & -0.11 & This study \\
\hline \multirow{3}{*}{$\begin{array}{l}\text { Mexico }\left(M_{w} 8.0\right) \\
(1985.09 .19)\end{array}$} & 0.084 & 277 & -0.089 & Gu 1996 \\
\hline & 0.086 & 231.46 & -0.085 & Chao and Gross 1987 \\
\hline & 0.101 & 232.97 & -0.004 & This study \\
\hline \multirow{2}{*}{$\begin{array}{l}\text { Sumatra }\left(M_{w} 9.0\right) \\
(2004.12 .26)\end{array}$} & 2.32 & 127 & -6.8 & Chao and Gross 1987 \\
\hline & 2.04 & 145 & -0.48 & This study \\
\hline
\end{tabular}

Table 2

\begin{tabular}{|c|c|c|c|c|c|c|c|}
\hline \multicolumn{8}{|c|}{ (a) Earthquake parameters used in different research. } \\
\hline Events & Depth $(\mathbf{k m})$ & Lat $\left({ }^{\circ}\right)$ & Lon $\left({ }^{\circ}\right)$ & $\begin{array}{c}\text { Moment } \\
\left(10^{29} \mathrm{dyn} \cdot \mathrm{cm}\right)\end{array}$ & Strike $\left(^{\circ}\right)$ & Dip $\left({ }^{\circ}\right)$ & Slip $\left({ }^{\circ}\right)$ \\
\hline Chile $\left(M_{w} 9.5\right)$ & 25 & -38.5 & 285.5 & 27 & 170 & 10 & 80 \\
\hline Alaska (M 9.2) & 50 & 61.0 & 213.0 & 7.5 & 114 & 9 & 270 \\
\hline Sumba $\left(\mathrm{M}_{\mathrm{w}} 8.3\right)$ & 43 & -11.1 & 118.5 & 0.36 & 260 & 17 & 24 \\
\hline $\operatorname{Mexico}\left(\mathrm{M}_{\mathrm{w}} 8.0\right)$ & 21 & 18.2 & 259.0 & 0.11 & 301 & 18 & 105 \\
\hline Sumatra $\left(\mathrm{M}_{\mathrm{w}} 9.0\right)$ & 29 & 3.1 & 94.3 & 4.0 & 329 & 8 & 110 \\
\hline \multicolumn{8}{|c|}{ (b) Result of polar motion and $\Delta$ LOD using different fault models and comparison with Gross' (2011) resul } \\
\hline & & \multicolumn{3}{|c|}{ amplitude (mas) } & \multicolumn{3}{|c|}{$\Delta \mathrm{LOD}(\mu \mathrm{s})$} \\
\hline \multicolumn{2}{|l|}{ Mean fault } & \multicolumn{3}{|c|}{5.35} & \multicolumn{3}{|c|}{1.12} \\
\hline \multicolumn{2}{|l|}{240 sub-fault } & \multicolumn{3}{|c|}{4.26} & \multicolumn{3}{|c|}{-0.49} \\
\hline \multicolumn{2}{|l|}{ Gross (2011) } & \multicolumn{3}{|c|}{5.67} & \multicolumn{3}{|c|}{-1.8} \\
\hline
\end{tabular}

it can be computed more simply than the amplitude; so, it will not be examined in this study. It can be predicted that the amplitude of polar motion caused by earthquakes is independent upon the longitude where earthquake occurs if we consider a SNREI Earth model or a rotating symmetrical Earth. This subsection presents derivation of the mathematical expression to prove this conclusion.

The excitation function of polar motion is given in the section above, where the excitation function's modulus is the amplitude of polar motion, so the expression of the amplitude of polar motion is written as the following.

$|\psi|=\frac{1.61}{C-A}\left(\Delta I_{13}^{2}+\Delta I_{23}^{2}\right)^{1 / 2}$
Based on Dahlen's analytical expression of inertia moments, by taking the change of inertia moments into Eq. (13), we can obtain the following expression.

$$
\begin{aligned}
& |\psi|=\frac{1.61 M_{0}}{C-A}\left[\left(\Gamma_{1} a_{1}+\Gamma_{2} a_{3}\right)^{2} \sin ^{2} 2 \phi+\Gamma_{1}^{2} a_{2}^{2} \cos ^{2} \phi\right. \\
& \left.+\Gamma_{3}^{2}\left(a_{4}^{2} \cos ^{2} 2 \phi+a_{5}^{2} \sin ^{2} \phi\right)+\Gamma_{3} a_{4} \sin 4 \phi\left(\Gamma_{1} a_{1}+\Gamma_{2} a_{3}\right)\right]^{1 / 2}
\end{aligned}
$$

Equation (14) shows that it involves all of the parameters save for the longitude. This fact means that the amplitude is independent upon longitude; an earthquake happening at an arbitrary longitude will produce the same change in the Earth's rotation. 


\section{SIMULATION OF EFFECTS OF EARTHQUAKE PARAMETERS ON EARTH ROTATION CHANGE}

As Table 1 shows, previous studies only computed the co-seismic rotation change and discussed the relationship between the magnitude and rotation change. However, the effect of different earthquake parameters on rotational change is still not discussed. This section presents a numerical simulation of how latitude, azimuth angle, and dip angle affect the polar motion and $\Delta$ LOD based on the theoretical scheme presented above.

To discuss the latitude effect is equivalent to answering how the co-seismic rotation changes with the latitude change. For this purpose, we consider a vertical strike slip fault model with magnitude $\mathrm{M}_{\mathrm{w}} 9.0$ and depth of $20 \mathrm{~km}$ (Fig. 1a). Then we compute the rotation changes for seven latitudes, as shown in Fig. 2. We also compute rotation changes for different azimuths of $0^{\circ}-360^{\circ}$. The horizontal axis is the azimuth angle and the vertical axis is the amplitude of polar motion (Fig. 2a) and $\triangle$ LOD (Fig. 2b). Re- sults of different latitudes are represented by different color curves: $0^{\circ}, 15^{\circ}, 30^{\circ}, 45^{\circ}, 60^{\circ}, 75^{\circ}$ and $90^{\circ}$.

Figure 2 presents the changes in amplitude of polar motion and $\triangle \mathrm{LOD}$ change with the azimuth angle and the latitude. The curved shape appears periodically about the azimuth angle. At $\left[0^{\circ}, 180^{\circ}\right]$, the amplitude has a minimum peak at $45^{\circ}$ and $135^{\circ}$, and maximum at $0^{\circ}, 90^{\circ}, 180^{\circ}$. The $\triangle \mathrm{LOD}$ has a maximum value at $45^{\circ}$. It becomes 0 at $0^{\circ}, 90^{\circ}$. It also has a minimum value at $135^{\circ}$. The same behavior appears at $180^{\circ}$ and $360^{\circ}$. This phenomenon implies that if the fault strike is north/south or west/east, then the earthquake will excite the maximal polar motion, but not affect the day length. The amplitude will be minimal and $\triangle \mathrm{LOD}$ will be maximal if the fault strike is north/east or south/west. This characteristic is important to understand the co-seismic rotation change. It can explain the non-uniformity between polar motion and $\triangle \mathrm{LOD}$ caused by different earthquakes in Table 1.

Next we discuss how the dip angle affects the Earth's rotation. We choose a fault model with a magnitude of
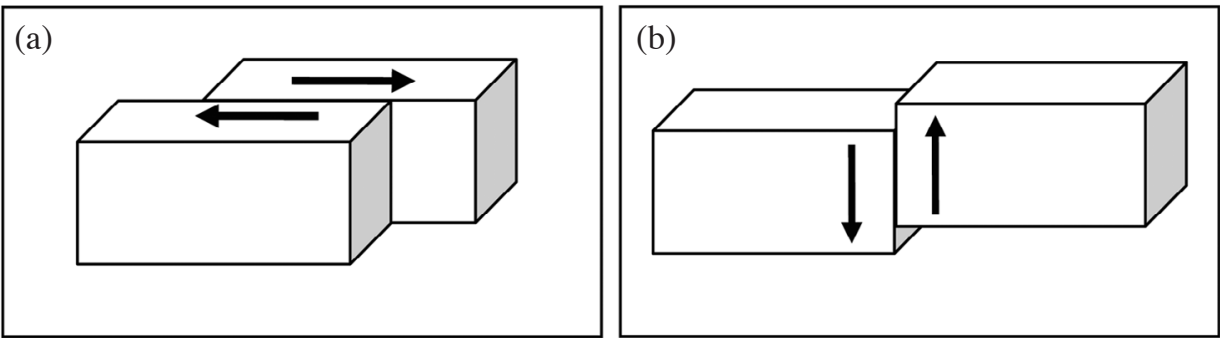

Fig. 1. The sketch map for different fault models used in the simulation: (a) vertical strike slip fault model with a depth of $20 \mathrm{~km}$ with changed azimuth, (b) the fault model with a depth of $20 \mathrm{~km}$, rake angle $90^{\circ}$, azimuth angle $45^{\circ}$ and changed dip angle.

(a)

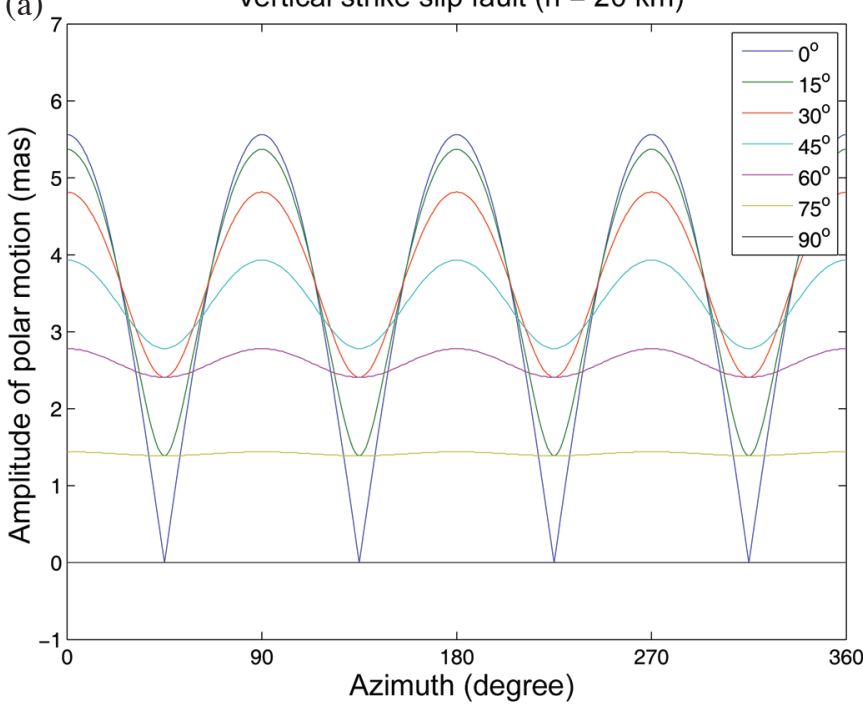

(b)

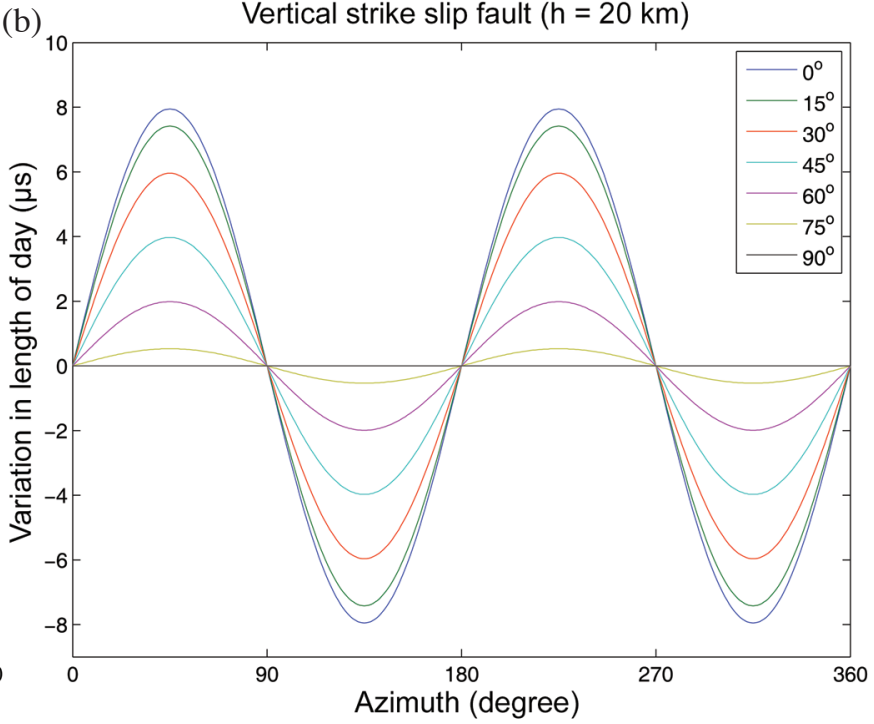

Fig. 2. Effects of azimuth and latitude on polar motion and $\triangle \mathrm{LOD}$ for a vertical strike slip fault. (a) Amplitude of polar motion (mas); (b) $\Delta L O D$ $(\mu s)$. 
$\mathrm{M}_{\mathrm{w}} 9.0$, depth of $20 \mathrm{~km}$, azimuth of $45^{\circ}$, and rake angle of $90^{\circ}$ (Fig. 1b). The range of the dip angle is $0^{\circ}-90^{\circ}$. Then we calculate the changes for different dip angles and different latitudes. Results are shown in Fig. 3.

Figure 3 shows that the change in the Earth's rotation is the same in the two hemispheres or symmetrical for the equator. However, the polar motion amplitude is symmetrical for dip angle $45^{\circ}$, which is the inflection point. It becomes 0 when the earthquake happens at either of the two poles or the equator. At the same time, the day length shows a large change at the poles and equator and it becomes 0 at dip angle of $45^{\circ}$. Figure 2 also shows that an earthquake speeds up the Earth's rotation at low or medium latitudes (less than $\pm 40^{\circ}$ ), but it slows rotation at high latitudes (greater than $\pm 40^{\circ}$ ).

\section{A CASE STUDY OF THE 2011 GREAT EAST JA- PAN EARTHQUAKE $\left(M_{W}\right.$ 9.0)}

As a case study, we compute the co-seismic rotation change produced by the 2011 Great East Japan earthquake. This event was the fifth largest earthquake since 1960, occurring in a subduction zone where the Pacific plate dives beneath the North American plate causing tsunamis. It also produced co-seismic rotation change. To study the effect of this earthquake on the Earth's rotation, we adopt the finite fault model used by Hayes (2011) and centroid moment solution for the 2011 Great East Japan earthquake from the USGS. To observe the effect of different fault models on rotation change, we also use different fault models in computations. Numerical results are presented in Table 2 and are compared with those of Gross (2011).
The effect on polar motion is 5.35 mas if we consider a homogeneous slipping model (i.e., a mean model). However, if we consider a fault slip distribution model, then the result is 4.26 mas. The latter is much smaller than the former. The $\triangle$ LOD shows even greater deviation in magnitude and changes in sign, which implies that an accurate fault slip model is important to calculate co-seismic Earth rotation change. The finer the slip model is, the more accurate the result is. Comparison to Gross' (2011) result reveals that the results are basically equal in magnitude, showing little difference. The reason for the difference is possibly attributed to the different methods. The method used by Gross is based on a normal mode method, while our method is based on Dahlen's elastic dislocation theory and summation for every sub-fault.

Then we discuss whether or not the co-seismic rotation change is detectable. Usually, the polar motion change observed by the GRACE (Gravity Recovery And Climate Experiment) and SLR (Satellite Laser Ranging) includes many geophysical effects such as co-seismic deformation, post glacial rebound, ice melting, and sea level change. Because GRACE data have been corrected for tide, ocean, and atmosphere signals, we use them to compute rotation change. In our computation, we use the GRACE data observed since 2003. We process the GRACE data using the least squares method, and observe the relation between polar motion and the second order stokes coefficients (Chao and Gross 1987), as shown in Fig. 4.

The red curve is the result obtained using the initial GRACE raw data. The green curve represents the result obtained using least-squares method which contains the constant term, the trend term, the year periodic term, and (a)

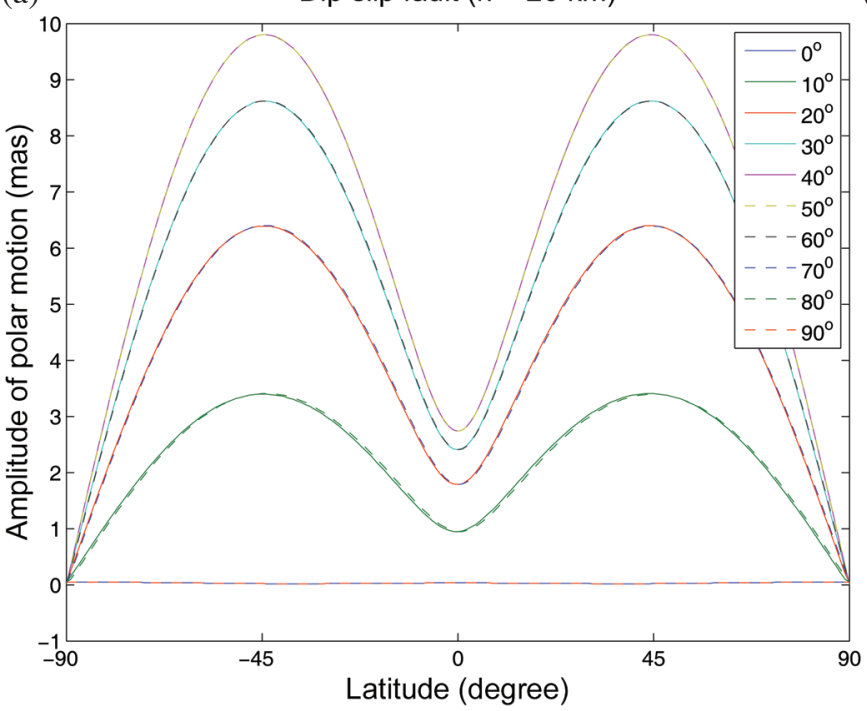

(b)

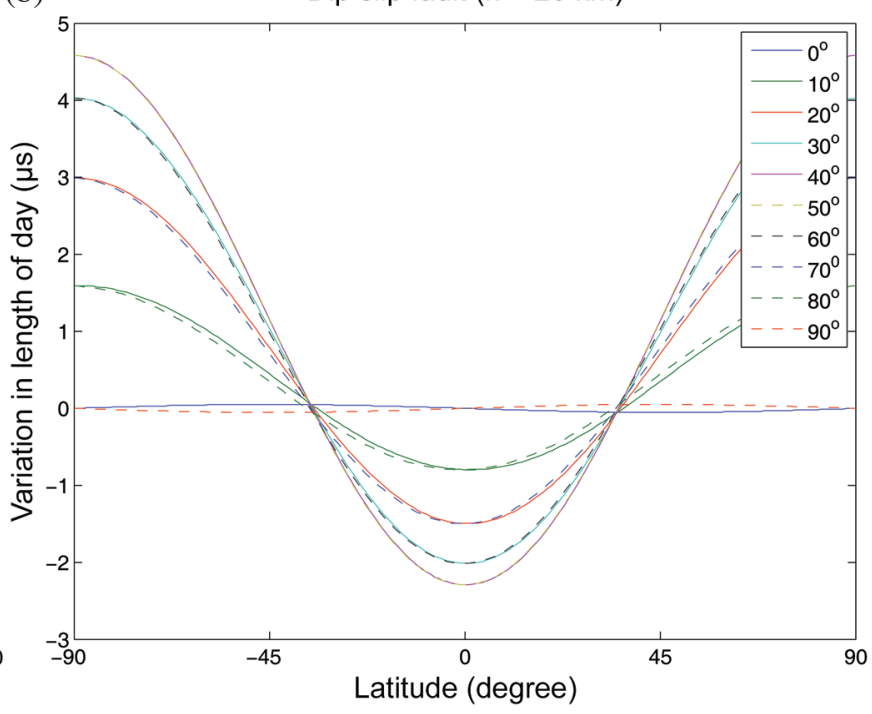

Fig. 3. Effects of latitude and fault dip angle on polar motion and change in the day length. (a) Amplitude of polar motion (mas); (b) $\Delta$ LOD ( $\mu$ s). 
Time series of changes of polar motion amplitude

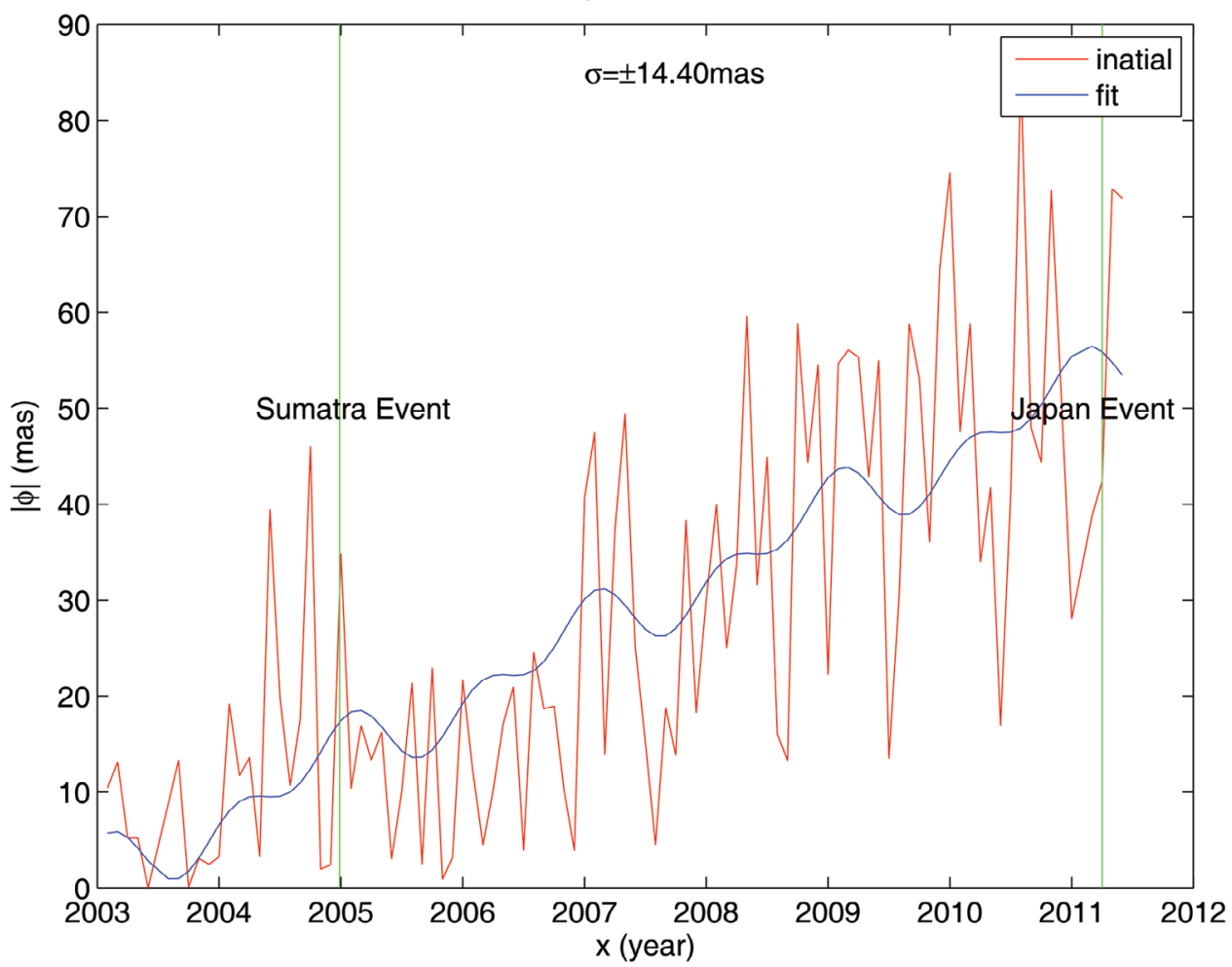

Fig. 4. Amplitude of polar motion observed by GRACE (red curve); the green curve is the fitted curve. Two blue curves show data for the 2004 Sumatra earthquake $\left(M_{w} 9.0\right)$ and the 2011 Great East Japan earthquake $\left(\mathbf{M}_{w} 9.0\right)$.

the half-year periodic term. Figure 3 shows that the initial GRACE data are influenced strongly by noise and show greater deviation than the fitting curve. The standard deviation of the polar motion change observed by GRACE is 14.43 mas. However, the magnitude of polar motion by large earthquakes is several mas, which is less than the standard deviation of the GRACE data. Therefore, it might be concluded that the co-seismic polar motion is difficult to detect because of noise or other geophysical effects. However, it would be possible for GRACE to detect the co-seismic polar motion induced by the 1960 Chile earthquake because the magnitude of the polar motion change by this earthquake is theoretically as much as 20 mas. In addition, considering the effect of earthquake on rotation change discussed above, if the earthquake occurs at the equator, then the azimuth angle and the dip angle are $45^{\circ}$, respectively, and the magnitude exceeds 9.0 , the rotation change is completely detectable by GRACE, at least by GRACE Follow-on.

\section{CONCLUSION AND DISCUSSION}

(1) We calculated the co-seismic Earth rotation change for several typical great earthquakes since 1960 using Dahlen's theory which approximates the Earth as a homogeneous sphere. The obtained numerical results agree well with the results obtained using theories based on more realistic Earth models that have been developed subsequent to the publication of Dahlen's method. This indicates Dahlen's theory gives good approximations for computation of co-seismic rotation changes.

(2) We proved that the amplitude of polar motion is independent of the longitude at which earthquakes occur. Results show that an earthquake occurring at an arbitrary longitude will produce the same polar motion change.

(3) For the same type of earthquake, the rotation change appears to have a large magnitude at low latitude. The rotation change decreases as latitude increases. The rotation change is symmetrical for the two hemispheres.

(4) The simulation results indicate that the co-seismic rotation change is symmetrical for a dip angle of $45^{\circ}$, i.e., when the dip angle decreases, the amplitude of polar motion decreases and the $\triangle \mathrm{LOD}$ increases. The maximum co-seismic change occurs at two poles and the equator.

(5) It is important to use detailed fault models when studying the rotation change because it is sensitive to fault models.

Finally, it should be pointed out that the investigation and discussion presented above are based on Dahlen's theory because of its simplicity. However, because Dahlen's theory is based upon a point source and because it cannot 
reflect a realistic distribution of fault slipping, the numerical results are regarded as bearing great uncertainty. This theory adopted a very simple Earth model, and it is only taken as a homogeneous sphere instead of layer structures. It means that Dahlen's theory gives a good approximation but not enough if the detailed fault slip distribution is considered; it is also expected that some inaccurate result is obtained if more realistic earth model is considered, such as layered structure, etc. Those two points can be improved using a reasonable dislocation theory and fault slipping model. Sun and Okubo (1993) and Sun et al. (2009) presented a spherical dislocation theory incorporating layer structures and earth's curvature effect. Considering the fault slipping model, it will give a higher precision. Therefore, our future studies will be devoted to computing the co-seismic ratio change using spherical dislocation with a more realistic SNREI Earth model.

Acknowledgements The authors are grateful to the editor (Kosuke Heki) and two anonymous reviewers for their helpful and constructive comments which greatly improved the paper. This study was financially supported by the CAS/ CAFEA International Partnership Program for Creative Research Teams (No. KZZD-EW-TZ-19) and by National Nature Science Foundation of China (Grant No. 41174063).

\section{REFERENCES}

Chao, B. F. and R. S. Gross, 1987: Changes in the Earth's rotation and low-degree gravitational field induced by earthquakes. Geophys. J. Int., 91, 569-596, doi: 10.11 11/j.1365-246X.1987.tb01659.x. [Link]

Chao, B. F., W. P. O'Connell, A. T. C. Chang, D. K. Hall, and J. L. Foster, 1987: Snow load effect on the Earth's rotation and gravitational field, 1979-1985. J. Geophys. Res., 92, 9415-9422, doi: 10.1029/JB092iB09p09415. [Link]

Dahlen, F. A., 1968: The normal modes of a rotating, elliptical Earth. Geophys. J. Int., 16, 329-367, doi: 10. 1111/j.1365-246X.1968.tb00229.x. [Link]

Dahlen, F. A., 1971: The excitation of the Chandler wobble by earthquakes. Geophys. J. Int., 25, 157-206, doi: 10. 1111/j.1365-246X.1971.tb02336.x. [Link]

Dahlen, F. A., 1973: A correction to the excitation of the Chandler wobble by earthquakes. Geophys. J. R. Astr. Soc., 32, 203-217, doi: 10.1111/j.1365-246X.1973.tb 06527.x. [Link]

Degryse, K. and V. Dehant, 1996: Are earthquakes responsible for the excitation of the FCN and/or of the FICN? Phys. Earth Planet. Inter., 94, 133-143, doi: 10.1016/0031-9201(95)03078-6. [Link]

Gross, R. S., 1986: The influence of earthquakes on the Chandler wobble during 1977-1983. Geophys. J. Int., 85, 161-177, doi: 10.1111/j.1365-246X.1986tb05176. x. [Link]

Gross, R. S., 2010: Chilean quake may have shortened Earth days, available at http://www.nasa.gov/topics/earth/ features/earth-20100301.html.

Gross, R. S., 2011: Japan quake may have shortened Earth days, moved axis, available at http://www.jpl.nasa. gov/news/news.php?release $=2011-080$.

Gross, R. S. and B. F. Chao, 2006: The rotational and gravitational signature of the December 26, 2004 Sumatran earthquake.Surv. Geophys., 27, 615-632, doi: 10.1007/ s10712-006-9008-1. [Link]

Gu, Z. N., 1996: The study of excitation of the earthquake to Earth's rotation. Earth Moon Planet, 74, 35-47, doi: 10.1007/BF00118720. [Link]

Hayes, G., 2011: Finite fault model updated result of the March 11, $2011 \mathrm{M}_{\mathrm{w}} 9.0$ earthquake offshore Honshu, Japan. Available at http://earthquake.usgs.gov/earthquakes/eqinthenews/2011/usc0001 xgp/finite fault. php.

Kobayashi, Y. and K. Heki, 2012: On the polar motion excitations by recent mega-thrust earthquakes. J. Geod. Soc. Japan, 58, 89-93. (in Japanese)

Lambeck, K., 1980: The Earth's Variable Rotation: Geophysical Causes and Consequences (Cambridge Monographs on Mechanics), Cambridge University Press, London, $460 \mathrm{pp}$.

Mansinha, L. and D. E. Smylie, 1967: Effect of earthquakes on the Chandler wobble and the secular polar shift. $J$. Geophys. Res., 72, 4731-4743, doi: 10.1029/JZ072i01 8p04731. [Link]

Nilsson, T., J. Bohm, and H. Schuh, 2010: Impacts of the 2010 Chile earthquake on earth rotation. AGU Spring Meeting, Foz do Iguau, Brazil, August 08-12, 2010.

O'Connell, R. J. and A. M. Dziewonski, 1976: Excitation of the Chandler wobble by large earthquakes. Nature, 262, 259-262, doi: 10.1038/262259a0. [Link]

Smith, M. L., 1977: Wobble and nutation of the Earth. Geophys. J. Int., 50, 103-140, doi: 10.1111/j.1365-24 6X.1977.tb01326.x. [Link]

Sun, W. and S. Okubo, 1993: Surface potential and gravity changes due to internal dislocations in a spherical earth - I. Theory for a point dislocation. Geophys. J. Int., 114, 569-592, doi: 10.1111/j.1365-246X.1993.tb 06988.x. [Link]

Sun, W., S. Okubo, G. Fu, and A. Araya, 2009: General formulations of global co-seismic deformations caused by an arbitrary dislocation in a spherically symmetric Earth model - Applicable to deformed Earth surface and space-fixed point. Geophys. J. Int., 177, 817-833, doi: 10.1111/j.1365-246X.2009.04113.x. [Link]

Trupin, A. S., M. F. Meier, and J. M. Wahr, 1992: Effect of melting glaciers on the Earth's rotation and gravitational field: 1965-1984. Geophys. J. Int., 108, 1-15, doi: 10.1111/j.1365-246X.1992.tb00835.x. [Link]

Wahr, J. M., 1982: The effects of the atmosphere and oceans on the Earth's wobble - I. Theory. Geophys. J. Int., 70, 349-372, doi: 10.1111/j.1365-246X.1982.tb04972.x. [Link] 